\title{
A Possible Explanation for Non-responders, Responders and Super- responders to Biologics in Severe Asthma
}

\author{
Michael E. Hyland ${ }^{1,2 *}$, Matthew Masoli2,3, Joseph W. Lanario ${ }^{1,2}$ and Rupert C. Jones ${ }^{4}$ \\ ${ }^{1}$ School of Psychology, University of Plymouth of Plymouth, Plymouth, UK; ${ }^{2}$ University Hospitals Plymouth NHS Trust, Plymouth, UK; \\ ${ }^{3}$ Royal Devon and Exeter Hospital, Exeter, UK; ${ }^{4}$ Peninsula School of Medicine and Dentistry, University of Plymouth, UK
}

\begin{abstract}
Response to biologic therapies in severe asthma is variable, with patients being either nonresponders, responders or super-responders. There is currently no explanation for this variation in response. If asthma-specific inflammatory pathways are part of a wider network of pathogenic mechanisms (including systemic inflammation), then the state of this wider network could either help or hinder the effect of the biologic. People with severe asthma are often polysymptomatic with a variable frequency of nonrespiratory symptoms. Application of existing network theory would predict that high systemic inflammation, measurable by the frequency of nonrespiratory symptoms, should decrease the effectiveness of biologics, a prediction consistent with the limited existing data. A detailed examination of the relationship between biologic response and the frequency or profile of nonrespiratory symptoms would provide a testable prediction of this hypothesis. The clinical presentation of super-responders is consistent with biologics sometimes having a positive effect on the pathology (level of dysregulation) in a network system. If that were the case, then network theory predicts the possibility of a short-term increase in nonrespiratory symptoms prior to the improvement reported by super-responders. If biologics lead to less network dysregulation in some patients, then this raises the possibility of new applications for this therapy and of an improved response to biologics if lifestyle improvement is started prior to biologic therapy.
\end{abstract}

Biologic therapies target specific inflammatory pathways involved in the pathogenesis of asthma, and because they have a highly specific effect on the inflammatory pathways, they reduce the inflammation of asthma with comparatively few side effects. The global initiative for asthma (GINA) guidelines for severe and difficult to treat asthma provide the following guidance": "If available and affordable consider an-add on Type 2 targeted biologic for patients with exacerbations and eosinophilic and/or allergic biomarkers despite taking high dose ICS-LABA with or without daily OCS" [sic] p 23.

Countries follow this recommendation but the criteria for number of exacerbations and levels of biomarkers varies between countries because availability and affordability varies between countries. In practice, this means that the use of biologics differs between countries, but in all countries biologics are restricted to the more severe cases for reasons of cost and evidence. Patients

Keywords: Severe asthma; Responder; Network; Complexity; Biologic therapy; Outcome.

Abbreviation: GINA, global initiative for asthma.

Received: April 19, 2019; Revised: May 21, 2019; Accepted: May 31, 2019

*Correspondence to: Michael E. Hyland, School of Psychology, University of Plymouth of Plymouth, Plymouth, UK. E-mail: mhyland@plymouth.ac.uk

How to cite this article: Hyland ME, Masoli M, Lanario JW, Jones RC. A Possible Explanation for Non-responders, Responders and Super-responders to Biologics in Severe Asthma. Exploratory Research and Hypothesis in Medicine 2019;4(2):35-38. doi: 10.14218/ERHM.2019.00008. who are eligible according to the GINA criteria show a variable response to biologics. Expert opinion recommends a traffic light system, based on assigning patients to one of three groups: nonresponders, responders, and super-responders. ${ }^{2}$ The consensus from this group is that a number of clinical indicators should be used to allocate patients to one of these three response groups and that the assessment should be made by the clinician after 4 months. The group did not define a super-responder, but clinical experience shows that some patients report biologics to be life-changing, as their life-dominating asthma all but disappears.

The majority of eligible patients respond well to biologic treatment. Two large studies found that $82-83 \%$ of patients are responders to omalizumab and two smaller studies found that $76-77 \%$ of patients are responders to mepolizumab. ${ }^{3-6}$ Although, the criteria for responder versus nonresponder differed between these studies, reflecting the consensus statement that several indicators can be used. ${ }^{2}$

There are limited explanations for the variable response to biologics. In those patients who meet the criteria for raised eosinophilic or allergic biomarkers, higher levels of baseline biomarkers are associated with better response to a biologic according to some studies but not others. ${ }^{6-10}$ This mechanism, however, cannot explain the substantial variation in response between non-responders and super-responders that is observed in clinical practice. Greater severity, measured by recent past exacerbations also predict better outcome measured by exacerbations,, 911 though this may be due to regression to the mean. Other baseline characteristics when used as predictors show more variable results. In one study, age, obesity, 


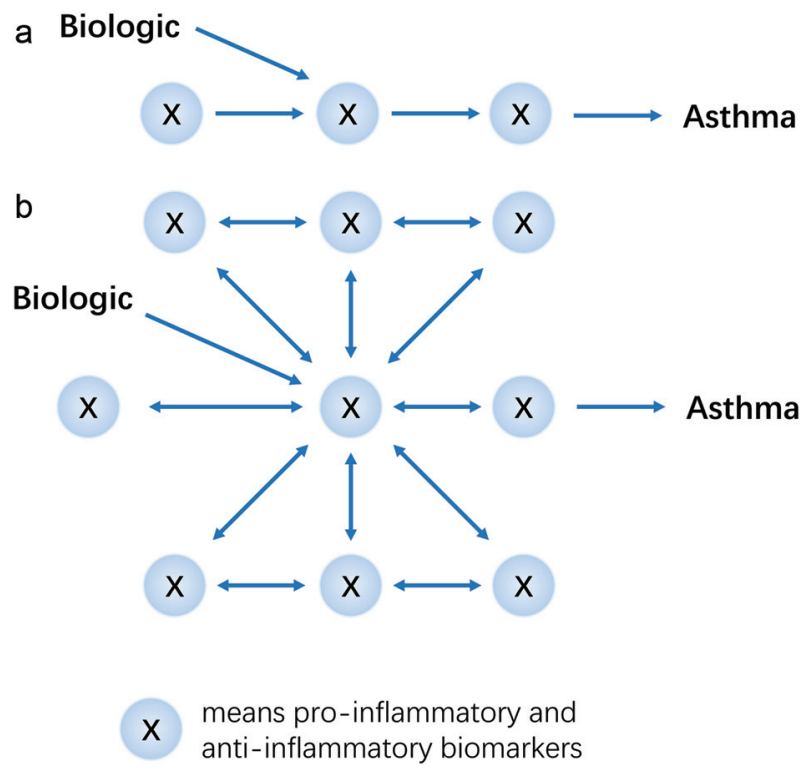

Fig. 1. Two models of how a biologic affects asthma. $A$ is a linear model. $B$ is a network model.

comorbidities, smoking habits, nasal polyps, and allergic polysensitization were associated with reduced response to omalizumab. ${ }^{12}$ However, in another study, obesity and cardiovascular disease were not found to be predictive of response to omalizumab. ${ }^{4}$ One study found that sex, body mass index, smoking history, allergies, and baseline level of eosinophils did not predict treatment response to mepolizumab. ${ }^{6}$ Genetic markers have also failed to predict outcome to mepolizumab, ${ }^{13}$ but there is preliminary evidence that exhaled breath analysis can predict outcome to this drug. ${ }^{14}$

The current theoretical model for the action of biologics can be represented by a linear causal sequence of an inflammatory pathway (Fig. 1a). According to this model, differences in response can be explained only in terms of the specific inflammatory mechanismand based on predictor studies, there is little evidence that this is the case. The proposed theoretical model expands this sequence by proposing that the target molecule is part of a causal network of other inflammatory markers or substances that have reciprocal causal relations. It is the state of this causal network that then determines the response of the target molecule to biologics, see Figure 1.

The hypothesis that several pathogenic mechanisms have a network architecture has been proposed elsewhere, as networks provide an explanation for otherwise difficult to explain symptomatology of functional disorders. ${ }^{15}$ Networks are highly sensitive to the rate of change on the simultaneous causal relations that occur between the nodes of the network and have emergent properties that are best understood in terms of algorithms or programs. ${ }^{16}$ If multiple pathogenic, symptom-causing mechanisms were connected to form a network architecture, then this would enable the body to adapt in ways that are more complex than previously thought. That is, the body would have the adaptive capability exhibited by a machine with artificial intelligence. ${ }^{15}$ The adaptive network theory suggests that the body's ability to adapt produces better regulation under most circumstances, but there are circumstances where it can produce dysregulation, ${ }^{17}$ which then has wide spread effects on numerous pathological mechanisms.

Severe asthma is often a polysymtomatic disease with numerous but variable non-respiratory symptoms, that include both psychological symptoms (e.g., fatigue, mental fog) as well as somatic symptoms (e.g., stomach pain, cold hands and feet). ${ }^{18}$ Many of these non-respiratory symptoms are a feature of systemic inflammation, but they are also a feature of medically unexplained symptoms that can be explained by a network of multiple symptomcausing mechanisms. ${ }^{15,17}$ It is therefore plausible that inflammatory pathways leading to asthma are also part of a wider network of pathogenic mechanisms that include systemic inflammation.

The systemic network-wide inflammation, of which the target molecule is part, could lock the target molecule into an inflammatory state, thereby reducing the effectiveness of the biologic treatment that would otherwise reduce inflammation. Systemic inflammation is an indicator of poorly functioning network, i.e. network dysregulation, and is associated with a number of non-respiratory symptoms. As different target molecules and different inflammatory pathways are embedded in different ways in the overarching inflammatory network, the relationship between biologic and nonrespiratory symptoms could be biologic-specific.

The first prediction is that:

More non-respiratory symptoms should predict worse response to a biologic. Different non-respiratory profiles may predict response to different biologics.

There is some existing evidence for this prediction. Depression reduces the effectiveness of biologics in arthritis, ${ }^{19}$ and comorbidity decreases the effectiveness of a biologic in asthma. ${ }^{12}$ However, there has been no systematic, theoretically-driven investigation of the hypothesis.

If an inflammatory pathway forms part of a wider network, then any therapy will affect not only the inflammatory pathway but also the wider network. It is possible for this effect to be either positive or negative. Glucocorticoids have a wide-ranging effect on the immune system, suppressing most cytokines, including antiinflammatory cytokines. ${ }^{20}$ These wide-ranging effects are more likely to have a dysregulatory effect on a finely tuned network, leading to gradual adaptation that is pathogenic. The finding that systemic steroids often have an initial energizing effect on patients but have long-term widespread adverse consequences is consistent with network functionality. Immediate change to the state of the network caused by the steroid is followed by gradual change to the activation rules (the causal connections between the nodes of the network) leading to adaptation. The long-term adaptation caused by steroids (in contrast to the short-term changes) include psychological symptoms (e.g., depression, fatigue) and somatic symptoms (bone fractures, skin thinning). All therapies carry some risk of adverse side effects because they affect mechanisms in addition to the target pathway, and it is the effect on these other mechanisms that could dysregulate the functioning of a biological network. However, because biologics have such a specific effect on the asthma inflammatory pathway, it is possible that these therapies can have a unique effect of reducing pathology in the network.

Because of the substantial benefit reported by super-responders, which is difficult to explain in terms of improved respiratory function, this leads to a second prediction:

Super-responders to biologic treatment experience benefit because there is a reduction in the distributed pathology of the network, i.e. reduced network dysregulation.

There is some evidence that biologic treatment reduces depression in patients with psoriasis, ${ }^{21,22}$ which is consistent with the hypothesis that biologics have this additional benefit. The role of the immune system in depression is well established, ${ }^{23}$ so it is plausible that anti-inflammatory therapy for the lung reduces depression, but depression is also a correlate of other somatic and psychological symptoms, the etiology of which is linked to the 


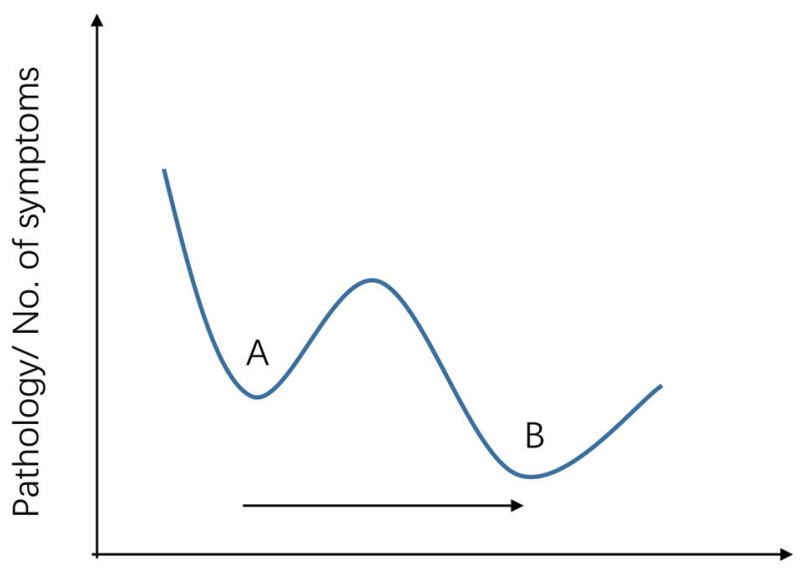

Therapeutic improvement

Fig. 2. Therapeutic improvement if the network is in a local minimum. Therapeutic improvement will lead to an initial increase in symptoms as the network moves from stage $A$ to stage $B$.

immune system.

Networks are complex systems of massive simultaneous causal connections. Depending on the initial state and depending on its inputs, networks can resolve into a particular stable state. The state of a network is determined by its historical state, such that different states, or local minima, are possible. ${ }^{24}$ Change from one local minimum to another (e.g., a lower local minimum) will involve the network moving initially in the opposite direction (Fig. 2). Let us suppose that networks vary in their level of dysregulation (high dysregulation $=$ poor health, low dysregulation $=$ good health), and that the level of dysregulation can be assessed by the number of non-respiratory symptoms. Let us suppose further that the network can adapt as a function of its inputs, therapeutic or pathogenic, so that dysregulation either increases or decreases. If the network is currently in one local minimum, then therapeutic change that leads the network to resolve into another, less pathogenic local minimum will require the network to move initially in a more pathogenic direction. So, if therapy requires the network to change from one stable state to another less dysregulated state, then there should be an initial increase in non-respiratory symptoms before those symptoms reduce below the original level.

Network adaptation is slow, so the increase and subsequent decrease in non-respiratory symptoms before their reduction could take a day or more. This leads to the following prediction:

Responders to biologic treatment (possibly only superresponders) may experience an increase in one or more non-respiratory symptoms for a period of time after starting (or receiving) biologic treatment, leading later to a reduction in those symptoms. Note, this prediction requires the network to resolves into local minima, and this is not yet established.

If the benefit experienced by super-responders is due to reduced dysregulation in a network of pathogenic mechanisms, this raises two possibilities, irrespective of the above prediction. First, the use of biologics earlier in the patient's history might prevent deterioration of non-respiratory symptoms. Second, biologics could have therapeutic benefit in other conditions, in particular for those patients with functional disorders who have raised levels of the target molecule. Although the number of patients benefiting so is likely to be small; a possible area for investigation is fibromyalgia as the non-respiratory symptoms of severe asthma have a similar profile to that of fibromyalgia. ${ }^{18}$

The recommendation is:

Other uses of biologics should be considered if biologics reduce non-respiratory symptoms in asthma.

As the pathogenic state of the network is affected by lifestyle, with health promoting lifestyle leading to less network pathology, ${ }^{13,25}$ then multicomponent lifestyle change interventions (exercise, psychology nutrition) provided with a narrative that engages patients and produces adherence (such as body reprogramming) could improve the effectiveness of biologics. ${ }^{26}$

The final prediction is:

Lifestyle improvement prior to biologic therapy should enhance the effectiveness of biologic therapy for severe asthma.

\section{Summary}

The number or profile of non-respiratory symptoms may predict response to biologics. Biologics may reduce non-respiratory symptoms, thereby explaining the substantial benefit reported by superresponders. Additional applications would follow if biologics were shown to reduce non-respiratory symptoms in asthma. Investigation of potential network effects should include measurement of change of non-respiratory symptoms over time. As it is theoretically possible that lifestyle interacts with biologics, lifestyle improvement prior to biologic therapy may improve response.

\section{Acknowledgments}

This research was supported by the National Institute for Health Research (NIHR) Collaboration for Leadership in Applied Health Research and Care South West Peninsula (NIHR CLAHRC South West Peninsula). The views expressed are those of the author(s) and not necessarily those of the NHS, the NIHR or the Department of Health and Social Care.

\section{Conflict of interest}

All four authors have received speaking fees from pharmaceutical companies. Their research program into severe asthma has been funded by non-promotional grants from industry.

\section{Author contributions}

MH developed the original idea using clinical information provided by MM, JL and RC, who then contributed to refinement of the theory. Initial drafting of the manuscript was done by MH. Critical revision of the article for important intellectual content was done by MM, JL and RJ.

\section{References}

[1] GINA (global initiative for asthma). Difficult to treat and severe asthma in adults and adolescents: a pocket guide. GINA, 2018. Available from: 
https://ginasthma.org/wp-content/uploads/2018/11/GINA-SAFINAL-wms.pdf.

[2] Buhl R, Humbert M, Bjermer L, Chanez P, Heaney LG, Pavord I, et al. Severe eosinophilic asthma: a roadmap to consensus. Eur Respir J 2017;49:1700634. doi:10.1183/13993003.00634-2017.

[3] Niven RM, Saralaya D, Chaudhuri R, Masoli M, Clifton I, Mansur AH, et al. Impact of omalizumab on treatment of severe allergic asthma in UK clinical practice: a UK multicentre observational study (the APEX II study). BMJ open 2016;6(8):e011857. doi:10.1136/bmjopen-2016-011857.

[4] Gibson PG, Reddel H, McDonald VM, Marks G, Jenkins C, Gillman A, et al. Effectiveness and response predictors of omalizumab in a severe allergic asthma population with a high prevalence of comorbidities: the Australian Xolair Registry. Intern Med J 2016;46(9):10541062. doi:10.1111/imj.13166.

[5] Kavanagh J, Green L, Fernandes M, Bolton M, d'Ancona G, Roxas C, et al. Assessment of early responder characteristics to mepolizumab in steroid-dependent eosinophilic asthma. Am J Resp Crit Care Med 2018;197:A1363.

[6] Drick N, Seeliger B, Welte T, Fuge J, Suhling H. Anti-IL-5 therapy in patients with severe eosinophilic asthma-clinical efficacy and possible criteria for treatment response. BMC Pulm Med 2018;18(1):119. doi:10.1186/s12890-018-0689-2.

[7] Ortega HG, Yancey SW, Mayer B, Gunsoy NB, Keene ON, Bleecker ER, et al. Severe eosinophilic asthma treated with mepolizumab stratified by baseline eosinophil thresholds: a secondary analysis of the DREAM and MENSA studies. Lancet Respir Med 2016;4(7):549-556. doi:10.1016/S2213-2600(16)30031-5.

[8] Kallieri M, Papaioannou Al, Papathanasiou E, Ntontsi P, Papiris S, Loukides $S$. Predictors of response to therapy with omalizumab in patients with severe allergic asthma-a real life study. Postgrad Med 2017;129(6):598-604. doi:10.1080/00325481.2017.1321945.

[9] FitzGerald JM, Bleecker ER, Menzies-Gow A, Zangrilli JG, Hirsch I, Metcalfe P, et al. Predictors of enhanced response with benralizumab for patients with severe asthma: pooled analysis of the SIROCCO and CALIMA studies. Lancet Respir Med 2018;6:51-64. doi:10.1016/ S2213-2600(17)30344-2.

[10] Humbert M, Taillé C, Mala L, Le Gros V, Just J, Molimard M, et al. Omalizumab effectiveness in patients with severe allergic asthma according to blood eosinophil count: the STELLAIR study. Eur Respir J 2018;51(5):1702523. doi:10.1183/13993003.02523-2017.

[11] Bousquet J, Wenzel S, Holgate S, Lumry W, Freeman P, Fox H. Predicting response to omalizumab, an anti-IgE antibody, in patients with allergic asthma. Chest 2004;125(4):1378-1386. doi:10.1378/ chest.125.4.1378.

[12] Sposato B, Scalese M, Milanese M, Masieri S, Cavaliere C, Latorre M, et al. Factors reducing omalizumab response in severe asthma. Eur J Intern Med 2018;52:78-85. doi:10.1016/j.ejim.2018.01.026.

[13] Condreay L, Chiano M, Ortega H, Buchan N, Harris E, Bleecker ER, et al. No genetic association detected with mepolizumab efficacy in severe asthma. Respir Med 2017;132:178-180. doi:10.1016/j. rmed.2017.10.019.

[14] van Bragt JJ, de Vries R, Sterk PJ, Maitland-van der Zee AH. Exhaled Breath Analysis for Prediction of Responders to Mepolizumab in Patients with Severe Asthma. In: Severe asthma: clinical and mechanistic studies. American Thoracic Society. 2019:A2673-A2673.

[15] Melidis C, Denham SL, Hyland ME. A test of the adaptive network explanation of functional disorders using a machine learning analy sis of symptoms. Biosystems 2018;165:22-30. doi:10.1016/j.biosystems.2017.12.010.

[16] Wolfram S. A new kind of science. Champaign, IL: Wolfram media, 2002.

[17] Hyland ME. The origins of health and disease. Cambridge: Cambridge University press, 2011.

[18] Hyland ME, Lanario JW, Jones RC, Masoli M. Evidence for similarity in symptoms and mechanism: the extra-pulmonary symptoms of severe asthma and polysymptomatic presentation of fibromyalgia. Inflammation, Immunity and Disease, in press.

[19] Artic Matcham F, Davies R, Hotopf M, Hyrich KL, Norton S, Steer S, et al. The relationship between depression and biologic treatment response in rheumatoid arthritis: An analysis of the British Society for Rheumatology Biologics Register. Rheumatology (Oxford) 2018;57(5):835-843. doi:10.1093/rheumatology/kex528.

[20] Brattsand R, Linden M. Cytokine modulation by glucocorticoids: mechanisms and actions in cellular studies. Alimentary Pharmacology \& Therapeutics 1996;10(Sup2):81-90. doi:10.1046/j.13652036.1996.22164025.x.

[21] Salame N, Ehsani-Chimeh N, Armstrong AW. Comparison of mental health outcomes among adults with psoriasis on biologic versus oral therapies: a population-based study. J Dermatolog Treat 2019;30(2):135-140. doi:10.1080/09546634.2018.1476654.

[22] Strober B, Gooderham M, de Jong EM, Kimball AB, Langley RG, Lakdawala $\mathrm{N}$, et al. Depressive symptoms, depression, and the effect of biologic therapy among patients in Psoriasis Longitudinal Assessment and Registry (PSOLAR). J Am Acad Dermatol 2018;78(1):70-80. doi:10.1016/j.jaad.2017.08.051.

[23] Trojan E, Chwastek J, Basta-Kaim A. A potential contribution of chemokine network dysfunction to the depressive disorders. Curr Neuropharmacol 2016;14(7):705-720. doi:10.2174/157015 9X14666160219131357.

[24] Ellis R, Humphreys GW. Connectionist psychology: A text with readings. Psychology Press, 1999.

[25] Hyland ME, Jeffery AN, Wilkin TJ. A biological, latent variable model of health (EarlyBird 68). Brain Behav Immun 2014;40:104-109. doi:10.1016/j.bbi.2014.02.018.

[26] Hyland ME, Hinton C, Hill C, Whalley B, Jones RC, Davies AF. Explaining unexplained pain to fibromyalgia patients: finding a narrative that is acceptable to patients and provides a rationale for evidence based interventions. $\mathrm{Br} J$ Pain 2016;10(3):156-161. doi:10.1177/2049463716642601. 